\title{
Complete chloroplast genome of Oncidium Gower Ramsey and evaluation of molecular markers for identification and breeding in Oncidiinae
}

\author{
Fu-Hui Wu¹, Ming-Tsair Chan+1, De-Chih Liao'1, Chen-Tran Hsu1', Yi-Wei Lee1, Henry Daniell2, Melvin R Duvall3 and \\ Choun-Sea Lin*1
}

\begin{abstract}
Background: Oncidium spp. produce commercially important orchid cut flowers. However, they are amenable to intergeneric and inter-specific crossing making phylogenetic identification very difficult. Molecular markers derived from the chloroplast genome can provide useful tools for phylogenetic resolution.

Results: The complete chloroplast genome of the economically important Oncidium variety Onc. Gower Ramsey (Accession no. GQ324949) was determined using a polymerase chain reaction (PCR) and Sanger based ABI sequencing. The length of the Oncidium chloroplast genome is $146,484 \mathrm{bp}$. Genome structure, gene order and orientation are similar to Phalaenopsis, but differ from typical Poaceae, other monocots for which there are several published chloroplast (cp) genome. The Onc. Gower Ramsey chloroplast-encoded NADH dehydrogenase (ndh) genes, except $n d h E$, lack apparent functions. Deletion and other types of mutations were also found in the ndh genes of 15 other economically important Oncidiinae varieties, except $n d h E$ in some species. The positions of some species in the evolution and taxonomy of Oncidiinae are difficult to identify. To identify the relationships between the 15 Oncidiinae hybrids, eight regions of the Onc. Gower Ramsey chloroplast genome were amplified by PCR for phylogenetic analysis. A total of 7042 bp derived from the eight regions could identify the relationships at the species level, which were supported by high bootstrap values. One particular 1846 bp region, derived from two PCR products (trnHGUG - psbA and trnFGAA-ndhJ) was adequate for correct phylogenetic placement of 13 of the 15 varieties (with the exception of Degarmoara Flying High and Odontoglossum Violetta von Holm). Thus the chloroplast genome provides a useful molecular marker for species identifications.

Conclusion: In this report, we used Phalaenopsis. aphrodite as a prototype for primer design to complete the Onc. Gower Ramsey genome sequence. Gene annotation showed that most of the ndh genes inOncidiinae, with the exception of $n d h E$, are non-functional. This phenomenon was observed in all of the Oncidiinae species tested. The genes and chloroplast DNA regions that would be the most useful for phylogenetic analysis were determined to be the $\operatorname{trnHGUG-psbA}$ and the trnFGAA-ndhJ regions. We conclude that complete chloroplast genome information is useful for plant phylogenetic and evolutionary studies in Oncidium with applications for breeding and variety identification.
\end{abstract}

\section{Background}

The Oncidiinae subtribe of the Orchidaceae family, consisting of about 70 closely related genera with over 1000 species, is divided into five alliances, with Oncidium as its largest genus [1]. From the perspective of cellular biology, ecology and morphology, Oncidium is the most diverse

\footnotetext{
* Correspondence: cslin99@gate.sinica.edu.tw

1 Agricultural Biotechnology Research Center, Academia Sinica, Taipei, Taiwan + Contributed equally

Full list of author information is available at the end of the article
}

genus in the Orchidaceae. Traditionally, the taxonomy of the Oncidiinae tribe is based on the morphology of the flower [2]; however, morphology is affected by environmental factors, and over time flower morphologies have evolved convergently. The positions of some species in the evolution and taxonomy of Oncidiinae are therefore difficult to identify. Accurate identification is further complicated by the ease with which Oncidiinae can be crossed intergenerically, as indicated by the 107 interge- 
neric hybrids reported [1] and the fact that more than 2200 hybrids (about $20 \%$ in the Oncidium group) have been re-distributed into other genera.

Different molecular marker techniques such as terminal restriction fragment length polymorphism (TRFL), arbitrarily primed polymerase chain reaction (AP-PCR), DNA amplification fingerprinting (DAF), and random amplification polymorphism DNA (RAPD)] are available to conduct genetic analyses by PCR and provide information about evolution that is useful for taxonomy. Tsai et al. [3] used 257 RAPD markers to investigate the relationships between 24 species of Oncidiinae, and found that the species could be separated into seven groups; however, Tsai and colleagues were unable to identify the more detailed relationships among these species.

Although there are three different genomes in plants, chloroplast DNA (cpDNA) is in many respects the genome of choice for taxonomic studies in orchids [2] as well as other species $[4,5]$. There are many advantages to using cpDNA for taxonomy and evolutionary research: (1) the size of cpDNA is small, with high copy number and simple structure; (2) when compared to the mitochondrial and nuclear genome, cpDNA gene content and arrangement are more conserved, making it easier to design primers and clone genes; (3) cpDNA is maternally inherited and thus without the genetic reassortment that interferes with the molecular phylogenetic relationships $[4,5]$.

The chloroplast genome is a circular chromosome of $120 \sim 220 \mathrm{~kb}$ that consists of two inverted repeats (IRa and IRb), a large single-copy region (LSC), and small singlecopy region (SSC). This conserved structure and sequence information provides a resource for primer design for other cpDNA sequencing by PCR [6]. This approach has been used for the sequencing of two bamboo cpDNA genomes [7]. As chloroplast genome of one member of the Orchidaceae family, Phalaenopsis aphrodite, has already been published [8], it is very useful to sequence complete cpDNA from another orchid, such as Oncidium using PCR.

The Chloroplast genome also has applications in plant biotechnology. Chloroplast genetic engineering offers a number of unique advantages, including high levels of transgene expression, multi-gene engineering in a single transformation event, transgene containment via maternal inheritance and a lack of gene silencing and position effects $[9,10]$. However, the lack of complete chloroplast genome sequences is still a major limitation to extending this technology. Additional information about the chloroplast genome would, thus, be of great value in advancing orchid biotechnology.

In this study, we designed primers based on the $P$. aphrodite cpDNA and used them to identify the cpDNA of Onc. Gower Ramsey, an important cut flower orchid.
Such primers were also used to investigate the $N A D H$ dehydrogenase (ndh) gene deletion patterns in 15 members of the Oncidiinae, and sequence amplified DNA regions to undertake phylogenetic analyses broadly across the angiosperms and at the species level.

\section{Methods}

\section{Plant materials}

Fifteen commercial Oncidiinae varieties were obtained from a grower (Yung Hsin Orchid nursery) in Taichung, Taiwan, including four Oncidium (Onc. Gower Ramsey, Gower Ramsey 'Lemon heart', Gower Ramsey 'Sunkiss', and Sweet Sugar 'Million Coins'), five Beallara (Bllra. Eurostar, Peggy Ruth Carpenter 'Morning Joy', Marfitch 'Howard Dream', Tahoma Glacier 'Sugar Sweet' and Smile Eri), two Odontoglossum (Odm. Margarete Holm and Violetta von Holm), two Odontocidium (Odcdm. Golden Gate, Odcdm. Wildcat 'Garfield'), one Degarmoara (Dgmra. Flying High) and one Zelenkocidium (Zelenkocidium Little Angel). These orchids were maintained in the greenhouse at Academia Sinica, Taipei, Taiwan, and vouchers specimens were deposited at the National Natural and Science Museum, Taichung, Taiwan. Leaves from these orchids were used in this study. Details of the parents of these species are shown in Figure 1.

\section{DNA purification, primer design and genomic PCR}

The PCR strategy for sequencing the chloroplast genome was adapted from Wu et al. [7]. For the chloroplast genomic PCR analysis, total genomic DNA from greenhouse-grown plants was isolated using a urea extraction buffer system [11]. The coding regions of the P. aphrodite chloroplast genome were used as the templates for primer design. A series of overlapping DNA fragments of 2 to $3 \mathrm{~kb}$ were amplified using specific primers (Additional file 1). The overlaps between adjacent PCR fragments were about $200 \mathrm{bp}$. The PCR amplification program consisted of 30 cycles of at $94^{\circ} \mathrm{C}$ for $30 \mathrm{~s}$, at $55^{\circ} \mathrm{C}$ for $30 \mathrm{~s}$ and at $72^{\circ} \mathrm{C}$ for $90 \mathrm{~s}$. The PCR products were sequenced. DNA sequencing was carried out with the Big-Dye Terminator Cycle Sequencing kit using an ABI Prism 3,700 DNA analyzer (Applied Biosystems, Foster City, CA). All gaps were filled by designing new primers on the basis of sequences obtained from PCR products (Additional file 1). The sequences were verified by comparison with the chloroplast genome of $P$. aphrodite using the VectorNTI AlignX software program (vers. 7.0; Invitrogen, Carlsbad, CA; parameters: overlap: 30; identity: 0.95; cutoff score: 40 ).

\section{Broad Phylogenetic analysis}

Analyses of 48 species were performed using the same 61 conserved protein-coding genes analyzed in previous studies [12-15]. This set of loci was assembled from the 


\begin{tabular}{llll}
\hline Genus & Variety & Ovary Parent & Pollen Parent \\
\hline Oncidium $^{1}$ & Gower Ramsey & Onc. Goldiana & Onc. Guinea Gold \\
& Gower Ramsey 'Lemon heart' & Onc. Goldiana & Onc. Guinea Gold \\
& Gower Ramsey 'Sunkiss' & Onc. Goldiana & Onc. Guinea Gold \\
& Sweet Sugar 'Million Coin' & Onc. Aloha ${ }^{2}$ & Onc. varicosum \\
Beallara & Eurostar & Bllra. Tahoma Glacier & Onc. schrodederianum \\
& Peggy Ruth Carpenter 'Morning Joy' & Bllra. Tahoma Glacier & Milt. Purple Queen \\
& Marfitch 'Howard Dream' & Mtssa. Charles ${ }^{3}$ & Oda. Fremar \\
& Tahoma Glacier 'Sugar Sweet' & Mtssa. Cartagena & Oda. Alaskan Sunset \\
& Smile Eri & Bllra. Tahoma Glacier & Oda. (Toroma X Ingera) \\
Odontoglossum & Margarete Holm & Odm. bictoniense & Odm. Hans koch \\
& Violetta. von Holm & Odm. bictoniense & Odm. Bic-ross \\
\hline Odontocidium & Golden Gate & Odm. bictoniense & Odcdm. Tiger Hambuhren \\
& Wildcat 'Garfield' & Odcdm. Rustic Bridge & Odcdm. Crowborough \\
\hline Zegarmoara & Flying High & Mtssa. Jet setter & Odm. McNabianum \\
\hline
\end{tabular}

Figure 1 Parents of 15 varieties of Oncidiinae. 'Yellow background: Oncidium; white: Beallara; blue: Odontoglossum; purple: Odontocidium; green: Degarmoara; red: Zelenkocidium. ${ }^{2}$ Onc. Aloha $=$ Onc. Goldiana $\times$ Onc. Star Wars. ${ }^{3}$ Mtssa Charles $=$ Brassia verrucosa $\times$ Milt. spectabilis.

aligned Nexus file for 45 species that is supplemental to the paper by Hansen et al. [[14]; available from http:// chloroplast.cbio.psu.edu/organism.cgi]. Also included were sequences from Lemna minor (GenBank accession NC 010109), Joinvillea plicata (GeneBank accessions

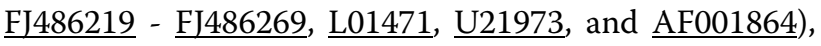
and Hordeum vulgare (NC_008590) to increase sampling among monocots and break up putative long branches. Gaps introduced by the alignment were excluded from phylogenetic analyses. Two phylogenetic methods were used-- maximum likelihood (ML), implemented in GARLI vers. 0.951-1 [16], and maximum parsimony (MP), implemented in PAUP* vers. 4.0b10 [17]. ML analyses were run under the general time reversible model, with all parameters estimated. A heuristic search of 100 random addition replicates was conducted for the MP analyses. Nonparametric bootstrap analyses were also performed with 100 (ML) or 1000 (MP) pseudoreplicates [18]. Ginkgo biloba was the specified outgroup for all analyses [14].

\section{Contig assembly and annotation}

VectorNTI Contig Express was used to assemble contigs (parameters: overlap: 30; identity: 0.95; and cutoff score: 40). The chloroplast genome was annotated using
DOGMA (Dual Organellar GenoMe Annotator) [19]. This program uses a FASTA-formatted input file of the complete genomic sequences and identifies putative protein-coding genes by performing BLASTX searches against a custom database of published chloroplast genomes. Both tRNAs and rRNAs were identified by BLASTN searches against the same database of chloroplast genomes. For genes with low sequence identity, manual annotation was performed after identifying the position of the start and stop codons, as well as the translated amino acid sequence, using the chloroplast/bacterial genetic code.

\section{Analysis of variability in ndh genes of 15 Oncidium varieties}

To investigate the $n d h$ genes of Oncidiinae, six cpDNA regions (trnF $F^{\mathrm{GAA}}-n d h J-n d h K-n d h C, \quad \operatorname{trn} R^{\mathrm{ACG}}-\operatorname{trn} N^{\mathrm{GUU}}$ ndhF-rpl32, ccsA-ndhD, psaC-ndhE-ndhG, ndhG-ndhI$n d h A-n d h H$ and $n d h B$ ) were obtained by a PCR approach from the 15 varieties as indicated in Methods (Accession no.: GU175359-GU175415, Additional file 2). The primer sequences, sequence size and sequence position in Onc. Gower Ramsey of these regions in Onc. Gower Ramsey are shown in Figure 2. 


\section{Phylogenetic analysis of 15 Oncidium varieties}

To investigate the phylogenetic relationships between Oncidiinae at the species level, eight cpDNA regions [intergene region (trnH $H^{\mathrm{GUG}}$ - $p s b A$, trnF $F^{\mathrm{GAA}}$ - $n d h J$, ycf1-trn$R^{\mathrm{ACG}}$ ) and coding regions ( $a c c D$, matK, $r b c L, r p o B$, and rpoC1)] were obtained by PCR from plastid DNA of the leaves of the 15 varieties as above (Accession no.: GQ915119-GQ915133; GU132947-132992; GU136249GU136275; GU175340-GU175358, Additional file 2). The primer sequences, sequence size and sequence position of these regions in Onc. Gower Ramsey are shown in Figure 2. Phylogenetics were conducted using MEGA4 (gap opening penalty: 15; gap extension penalty: 6.66; DNA weight matrix: IUB; transition weight: 0.5 ; negative matrix: off; and delay divergent cutoff: $30 \%$ ) [20]. The evolutionary history was inferred using the maximum parsimony, minimum evolution (ME), neighbor-joining (NJ) and unweighted pair-group method with arithmetic mean methods. In these four analyses, the bootstrap consensus tree was inferred from 1000 replicates [18]. Branches corresponding to partitions reproduced in < $50 \%$ bootstrap replicates were collapsed. The values of replicate trees in which the associated taxa clustered together in the bootstrap test (1000 replicates) are shown next to the branches [18].

\section{Results}

\section{Oncidium chloroplast genome sequencing}

The size of the Onc. Gower Ramsey chloroplast genome is $146,484 \mathrm{bp}$ (Figure 3). The genome includes a pair of IRs of 25,755 bp each, a SSC region of $12,650 \mathrm{bp}$, and a LSC region of 82,324 bp. The Onc. Gower Ramsey chlo- roplast genome contains 101 different genes, of which 16 are duplicated in the IR, giving a total of 133 genes. There are 29 distinct tRNAs, six of which are duplicated in the IR. Sixteen genes contain one or two introns, with six of the introns in tRNAs. Coding regions make up $49.94 \%$ of the chloroplast genome $(41.86 \%$ protein-coding genes, $8.08 \%$ RNA genes) and non-coding regions, which contain intergenic spacer (IGS) regions and introns, comprise $50.06 \%$. The overall GC and AT content of the chloroplast genome is $37.32 \%$ and $62.68 \%$, respectively. The gene order of Onc. Gower Ramsey cpDNA is similar to that of the orchid P. aphrodite (Figure 3). The rps 15 gene is not included in the IR. In contrast with the chloroplast genomes of Poaceae, Onc. Gower Ramsey contained introns in the clpP and rpoC1 loci and had intact copies of the $a c c D$, and $y c f 2$ genes, which are incomplete or entirely missing in Poaceae.

The broad phylogenetic analysis resulted in two trees, an ML tree with $-\operatorname{lnL}=412281.26$ (Figure 4) and an MP tree of 75,521 steps and 14,974 parsimony informative characters. The MP tree had a a consistency index (excluding uninformative characters) of 0.3649 and a retention index of 0.5997 (tree not shown). The topologies of the monocot subtrees were identical for the two analyses in which Oncidium was maximally supported as the sister of Phalaenopsis and the two orchids were united with $Y u c c a$, another representative of Asparagales, with maximum support.

Analysis of variability in ndh genes of 15 Oncidium species Six cp DNA regions (trnF ${ }^{\mathrm{GAA}}-n d h J-n d h K-n d h C$, trnR $R^{\mathrm{ACG}}$

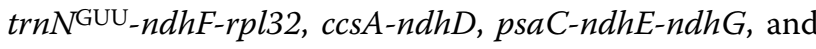

\begin{tabular}{|c|c|c|c|c|c|c|}
\hline & No. & Sequence & No. & Sequence & & \\
\hline $\operatorname{trn} F^{\mathrm{GAA}}-n d h J-n d h K-n d h C$ & 529 & TCGGGATAGCTCAGTTGGTA & 107 & GTTTCTGCTTCACGAATATG & 47650 & 1379 \\
\hline$t r n R^{\mathrm{ACG}_{-} t r n N^{\mathrm{GUU}}-n d h F-r p l 32}$ & 554 & ATTCGAACCTACGACCAGTCA & 1038 & TCCCTTTTTCTGACGAATTA & 107482 & 2196 \\
\hline $\operatorname{ccs} A-n d h D$ & 549 & TGAAATTGGTAGACACGCTGC & 1059 & ACCGAAGATTGTGTAGGTTG & 109333 & 2728 \\
\hline psaC-ndhE-ndhG & 1058 & TGCTCGGGAGAAGAATAATA & 1061 & TTTGTGGGAACCATAAATGT & 111849 & 1697 \\
\hline ndhG-ndhI-ndhA-ndhH & 1095 & TGAATACCAATTTGTTGAACG & 1917 & TCAAGTATTCCATTTCACCA & 113319 & 1828 \\
\hline$n d h B$ & 437 & TGATCTGGCATGTACAGAATG & 438 & AAAGAGGGTATCCTGAGCAA & 133250 & 2221 \\
\hline $\operatorname{trn} H^{\mathrm{GUG}}-p s b A$ & 460 & AAGCGTCCTGTAGTAAGAGGA & 476 & GGGAAACCACTGAAAATGAG & 145562 & 1426 \\
\hline$a c c D$ & 423 & TGGTTCAATTCAATGTTGTCT & 424 & ATTCAAGGGAAGGAAACCGT & 55778 & 1430 \\
\hline matK & 1785 & TCTAGCACACGAAAGTCGAAGT & 1784 & CGATCTATTCATTCAATATTTC & 1931 & 936 \\
\hline$t r n F^{\mathrm{GAA}}-n d h J$ & 529 & TCGGGATAGCTCAGTTGGTA & 107 & GTTTCTGCTTCACGAATATG & 47650 & 1379 \\
\hline$r b c L$ & 505 & AGGGAGGGACTTATGTCACCA & 506 & TATCTGGCTTATCCACTGGGT & 53528 & 1443 \\
\hline$r p o B$ & 986 & GCCTCTTGCTCATATCTCTC & 1078 & TGTGGAGCAATGAGGCATAA & 23345 & 1666 \\
\hline rpoCl & 893 & TATGAGTAGGCCCGCCAAA & 895 & TGGTCCTTACTGGGAACTTGA & 19989 & 1785 \\
\hline$y c f 1$-trnR $R^{\mathrm{ACG}}$ & 461 & AAAATCTTCGTAAACCGGGC & 554 & ATTCGAACCTACGACCAGTCA & 119956 & 1370 \\
\hline
\end{tabular}

Figure 2 Primers for Oncidiinae ndh gene and phylogenetic analysis. PPrimer sequences, annealing site of the forward primer in Onc. Gower Ramsey and the anticipated amplicon size (bp) are presented. ${ }^{2}$ Different background colors indicate different experiments; gray: $n d h$ gene identification; yellow: phylogenetic analysis. 


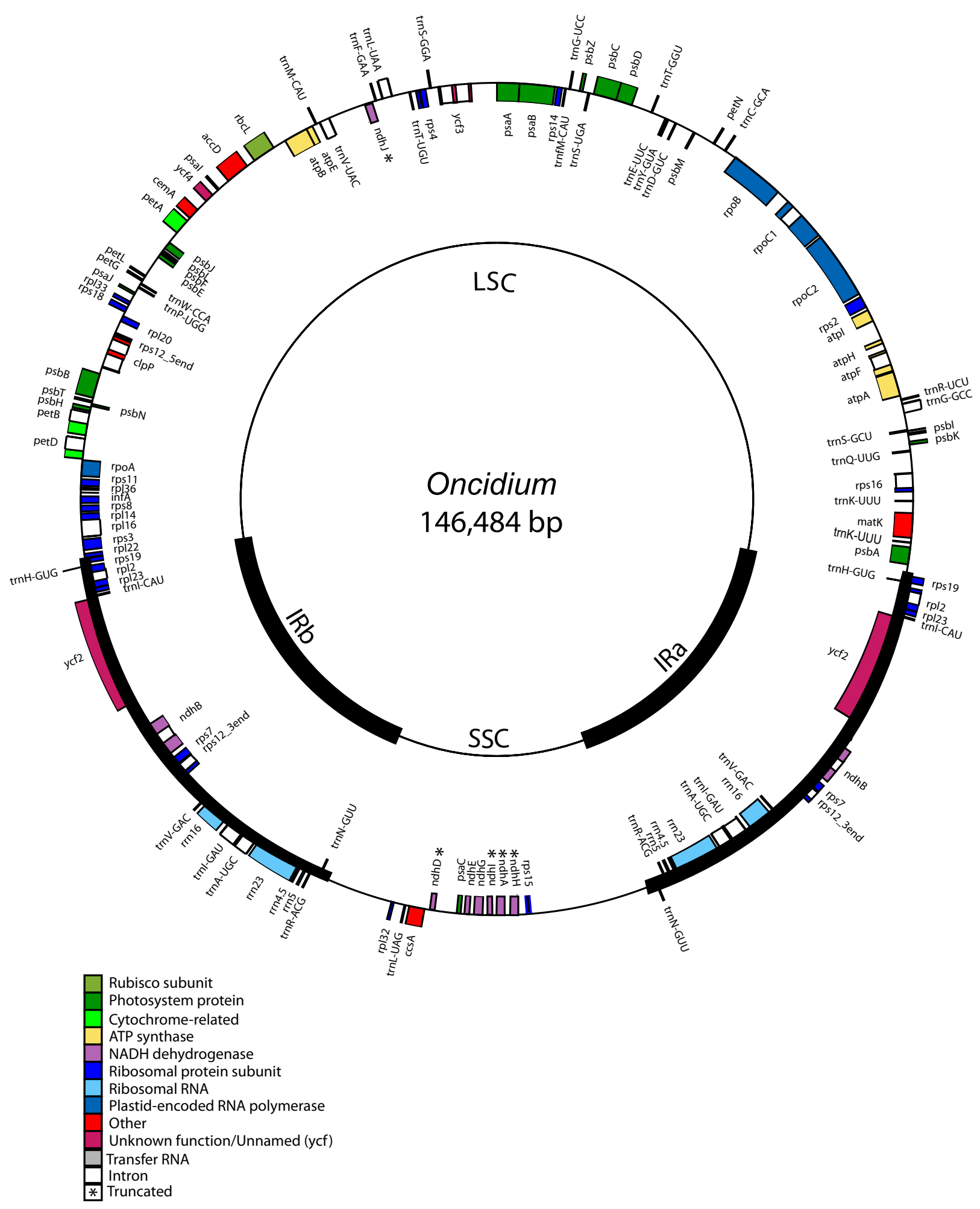

Figure 3 Gene map of Onc. Gower Ramsey chloroplast genome. The thick lines indicate the extent of the IRa and IRb, which separate the genome into SSC and LSC regions. Genes on the outside of the map are transcribed clockwise and genes on the inside of the map are transcribed counterclockwise. 


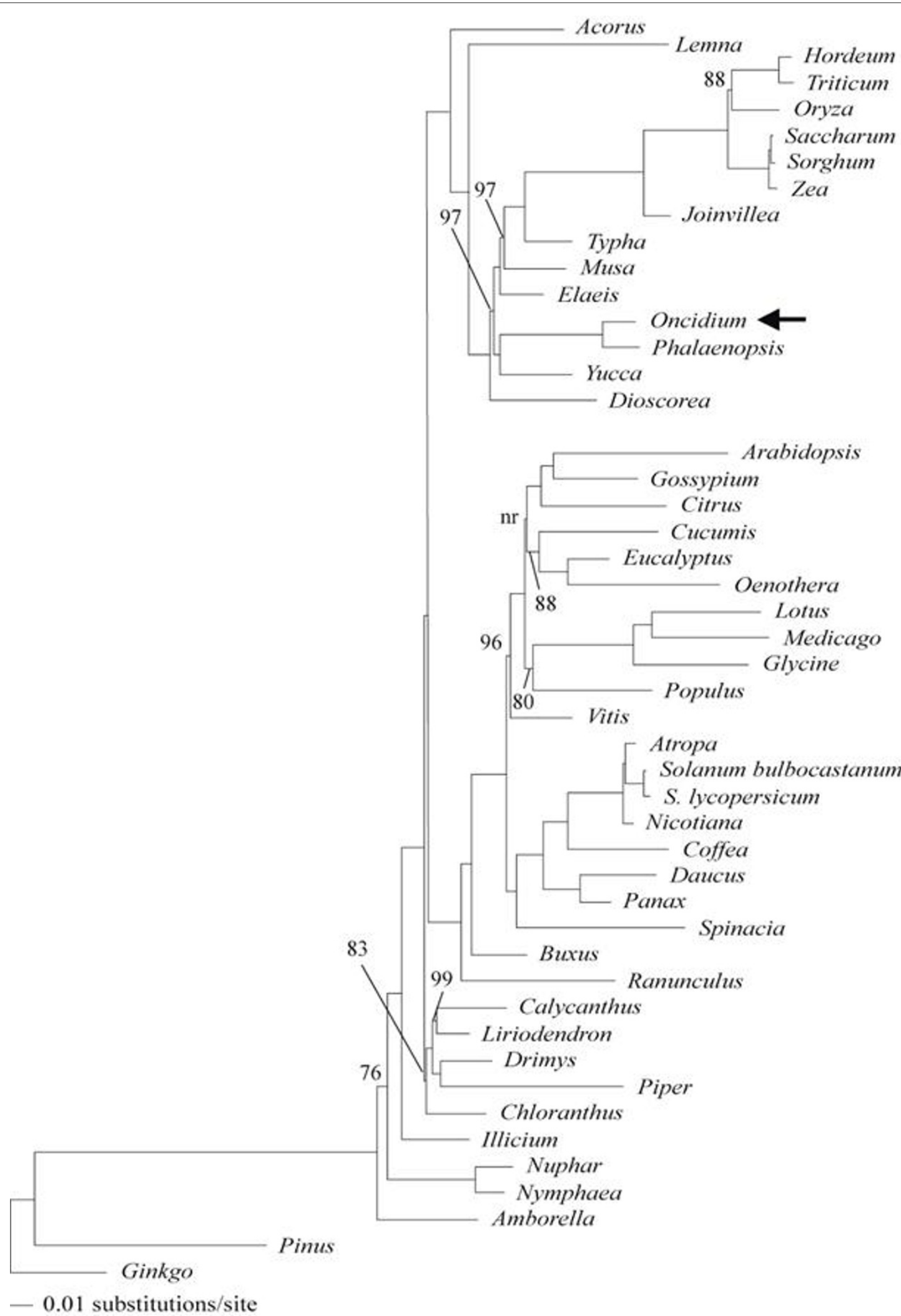

Figure 4 Maximum likelihood phylogram for 61 conserved protein-coding genes. All nodes have 100\% ML bootstrap support unless otherwise indicated. Horizontal branch lengths are proportional to the number of inferred substitutions/site along that branch. One node, marked "nr," was not resolved in the ML bootstrap consensus tree. The position of Oncidium in a clade of Asparagales is indicated with an arrow. 
$n d h G-n d h I-n d h A-n d h H$ and $n d h B)$ were obtained by PCR from total DNA of the leaves of the 15 varieties. Most of the $n d h$ genes in the 15 Oncidiinae varieties, with the exception of $n d h E$ in some species, had no function (Figure 5). In all 15 of the Oncidiinae varieties studied, the $n d h J$ gene was truncated (partial sequence remained) and the $n d h K$ gene was absent (no sequence exists). In $n d h C$, a frame shift occurred, creating a stop codon in the middle of the gene in all Oncidiinae, including Onc. Gower Ramsey, resulting from a $17 \mathrm{bp}$ deletion (Figure 6A, Figure 5).

The $n d h B$ of Oncidiinae does not function due to a stop codon in the first exon. To date, six orchid $n d h B$ genes, including $P$. aphrodite, have been cloned and published in the NCBI database. Two of them could translate putative functional $n d h B$ protein [Orchis rotundifolia (Accession no.: AY147484) and Coelogyne crisata (Accession no.: AY147475)]. There is also a frame shift in the second exon of Cypripedium passerinum (Accession no.: AY147479, AY147478.1). That of Odontoglossum crispum (AY834278) is only a partial sequence that could translate a putative $n d h B$ protein.

The $n d h F$ locus, which is located in the LSC-IRa junction, was absent in the Oncidiinae varieties. Notably, the nucleotide deletions in the $t_{r n R^{\mathrm{ACG}}-t r n N^{G U U}}-n d h F-r p l 32$ region were different between $P$. aphrodite and Oncidiinae (Figure 6B, Figure 5).

All of the 12 Oncidiinae $n d h D$ genes cloned here were truncated. The overall pattern of truncation can be classified into two types: a truncation occurring at the 3 '-end of $n d h D$ (as in the Bllra. varieties) and a truncation in the 5'end (in the rest of the clones tested) (Figure 6C, Figure 5).

The sequences of Onc. Gower Ramsey varieties, Onc. Sweet Sugar and Odm. Margarete Holm indicate that the translation capacity of $n d h E$ is retained in these species (Figure 5). Of the species with modified $n d h E$ genes, Odm. Violetta. von Holm, Odcdm. Golden Gate and Odcdm. Wildcat contained frame shifts; Dgmra. Flying High had a 30 bp deletion; and there were deletions of over $30 \mathrm{bp}$ in the four Beallara varieties (Figure 6D, Figure 5).

Although nine varieties had no deletions in the $n d h G$ genes, these varieties had three internal stop codons within $n d h G$, rendering $n d h G$ inactive (Figure 5). There was a deletion of about $250 \mathrm{bp}$ in the $n d h G$ gene of the Beallara species (Figure 6D, Figure 5).

The region encompassing $n d h I$ is the most complicated of the chloroplast $n d h$ gene regions. Generally, genes from the same genus had the same pattern (for example, see Oncidium and Beallara, Figure 6E, Figure 5). In the Onc. Gower Ramsey varieties, the $n d h I$ gene was partially deleted, and in the Beallara varieties, Zelenkocidium Little Angel and Odm. Violetta. von Holm, the $n d h I$ gene was completely absent.

Truncated $n d h A-n d h H$ genes still existed in most of the Oncidiinae species in this study. With the exception of frame shifts in Oncdm. Garfield $n d h A$ and Oncdm. Golden Gate $n d h H$, the other $n d h A$ through $n d h H$ genes in the other five genera all showed deletions of various types.

\begin{tabular}{|c|c|c|c|c|c|c|c|c|c|c|c|c|c|c|c|}
\hline & \multicolumn{4}{|c|}{ Oncidium ${ }^{1}$} & \multicolumn{5}{|c|}{ Beallara } & \multicolumn{2}{|c|}{ Odontoglossum } & \multicolumn{2}{|c|}{ Odontocidium } & \multirow{2}{*}{$\frac{\text { Degarmoara }}{\text { Dgmra. }}$} & \multirow{2}{*}{$\begin{array}{c}\text { Zelenkocidium } \\
\text { L. A. }\end{array}$} \\
\hline & $\mathrm{GR}^{2}$ & Sunkiss & L. H. & M. C. & E. star & M. J. & H. D. & S.S. & S.E. & М. н. & V. v. H. & G. G. & W. G. & & \\
\hline$n d h A$ & $\Delta^{3}$ & $\Delta$ & $\Delta$ & - & $\Delta$ & $\Delta$ & - & $\Delta$ & $\Delta$ & - & $\Delta$ & $\Delta$ & $\Delta$ & - & $\Delta$ \\
\hline$n d h B$ & 弥 & - & - & - & - & - & - & - & - & - & - & - & - & - & - \\
\hline$n d h C$ & $\Delta$ & $\Delta$ & $\Delta$ & $\Delta$ & $\Delta$ & $\Delta$ & $\Delta$ & $\Delta$ & $\Delta$ & $\Delta$ & $\Delta$ & $\Delta$ & $\Delta$ & $\Delta$ & $\Delta$ \\
\hline$n d h D$ & $\Delta$ & $\Delta$ & $\Delta$ & $\Delta$ & $\Delta$ & - & $\Delta$ & - & $\Delta$ & $\Delta$ & $\Delta$ & $\Delta$ & - & $\Delta$ & $\Delta$ \\
\hline$n d h E$ & O & O & 0 & O & $\Delta$ & $\Delta$ & $\Delta$ & - & - & O & $\Delta$ & $\Delta$ & $\Delta$ & $\Delta$ & - \\
\hline$n d h F$ & $\star$ & $\star$ & $\star$ & - & - & - & - & $\star$ & - & - & $\star$ & - & $\star$ & - & $\star$ \\
\hline$n d h G$ & $\Delta$ & $\Delta$ & $\Delta$ & $\Delta$ & $\Delta$ & $\Delta$ & $\Delta$ & - & - & $\Delta$ & $\Delta$ & $\Delta$ & $\Delta$ & $\Delta$ & - \\
\hline$n d h H$ & $\Delta$ & $\Delta$ & $\Delta$ & - & $\Delta$ & $\Delta$ & - & $\Delta$ & $\Delta$ & - & $\Delta$ & $\Delta$ & $\Delta$ & - & $\Delta$ \\
\hline$n d h I$ & $\Delta$ & $\Delta$ & $\Delta$ & - & $\Delta$ & $\Delta$ & - & $\Delta$ & $\Delta$ & - & $\Delta$ & $\Delta$ & $\Delta$ & - & $\Delta$ \\
\hline$n d h J$ & $\Delta$ & $\Delta$ & $\Delta$ & $\Delta$ & $\Delta$ & $\Delta$ & $\Delta$ & $\Delta$ & $\Delta$ & $\Delta$ & $\Delta$ & $\Delta$ & $\Delta$ & $\Delta$ & $\Delta$ \\
\hline$n d h K$ & $\star$ & $\star$ & $\star$ & $\star$ & $\star$ & $\star$ & $\star$ & $\star$ & $\star$ & $\star$ & $\star$ & $\star$ & $\star$ & $\star$ & $\star$ \\
\hline
\end{tabular}

Figure 5 Summary of $\boldsymbol{n d h}$ gene patterns in Oncidiinae. 'Different background colors indicate different genera; yellow: Oncidium, white: Beallara, blue: Odontoglossum, pink: Odontocidium, purple: Colmanara, green: Degarmoara, red:Zelenkocidium. ${ }^{2 G R: ~ G o w e r ~ R a m s e y, ~ S u n k i s s: ~ G o w e r ~ R a m s e y ~}$ 'Sunkiss', L. H.: Lemon heart, M. C.: Sweet sugar 'Million Coin', E. star: Eurostar, M. J.: Peggy Ruth Carpenter 'Morning Joy', H. D.: Marfitch 'Howard Dream', S. S.: Tahoma Glacier 'Sugar Sweet', S. E.: Smile Eri, M. H.: Margarete Holm, V. v. H.: Violetta. von Holm, G.G.: Golden Gate, W.G.: Wildcat 'Garfield', Dgmra:

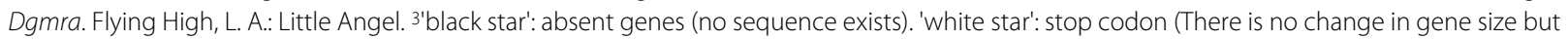
there are stop codons within coding sequences). 'black triangle': truncated genes (only partial coding sequences are observed). 'white triangle': frame shift (reading frame shifted or nucleotides deleted). 'white circle': functional protein., -: no PCR product obtained using the primers in Figure 2. 


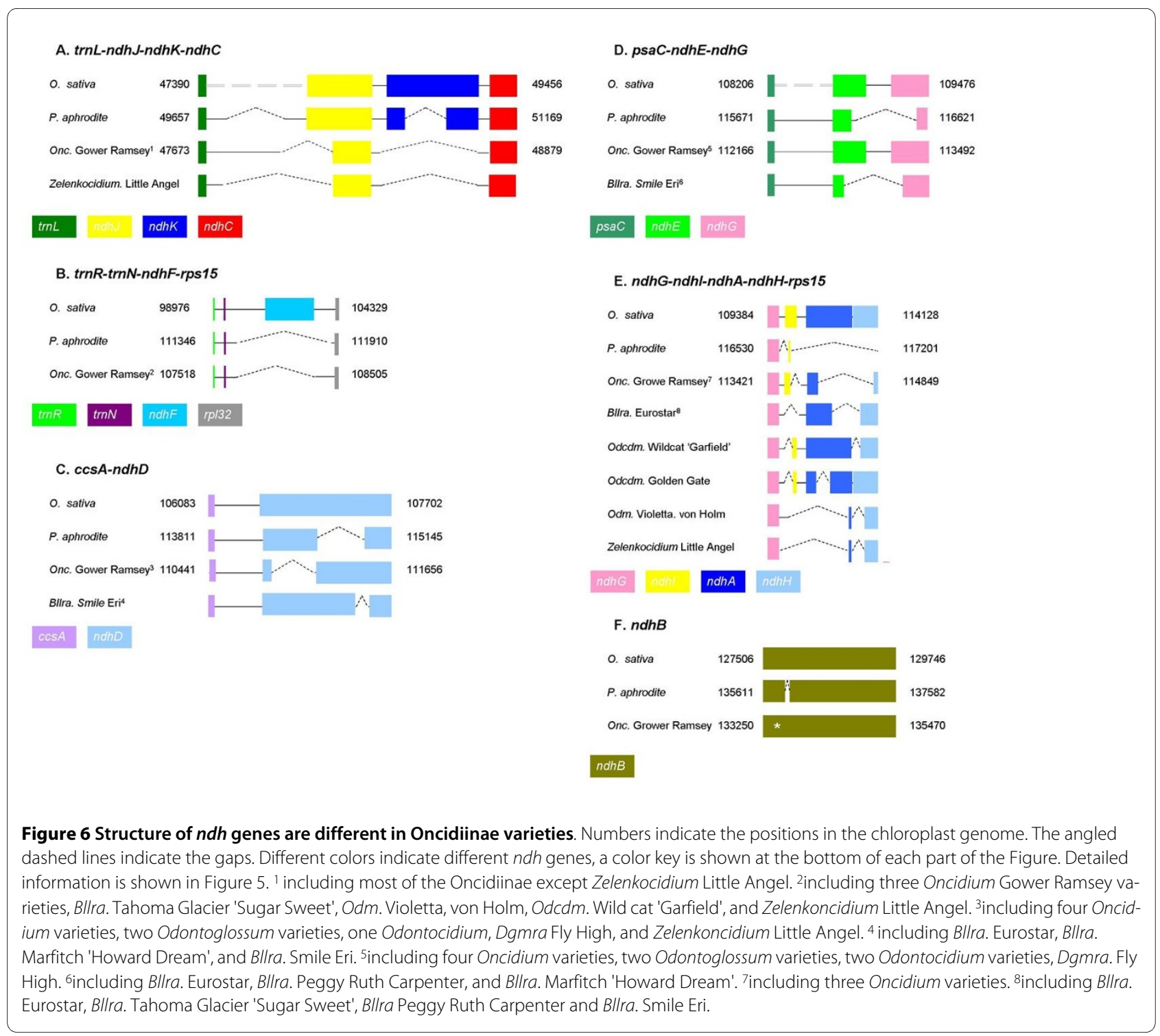

\section{Phylogenetic analysis of 15 Oncidium species}

Based on the amount of variation in the cpDNA and congruence with parent relationships, certain chloroplast regions were determined to be more useful than others. Because $r b c L$ is highly conserved; bootstrap scores are lower then $50 \%$ and are not useful for determining parent relationship (Additional file 3). Using the accD gene, only the species belonging to Beallara and Oncidium could be separated as the pattern and relationships among other species were not correlated with the parent relationship (Additional file 4).

In the mat $K$ region, the phylogenetic analysis of these sequences and 15 economic varieties gave results that correlated with parent relationship (Figure 7A). Therefore, we combined the most diverse cpDNA regions, $\operatorname{trn} H$ -psbA [21], matK and $t r n F^{\mathrm{GAA}}-n d h J$ [22], for phylogenetic analysis. The $t_{n} H^{\mathrm{GUG}_{-}} p s b A$ and $n d h J$ combination pro- vided the most similar results to those obtained from all eight cpDNA regions (Figure 7).

\section{Discussion}

Using PCR to sequencing Oncidium Gower Ramsey and phylogenomic applications

Although there are many methods for cp genome sequencing, PCR is one method that is easy and economical [7]. However, the gene content and order in monocot cp genomes is relatively diverse so that the use of the $P$. aphrodite as a template for primer design in this study was limiting. This was especially true in the $n d h$ gene regions where the deletion of $n d h$ genes in Oncidium is very different from that in $P$. aphrodite. Furthermore, when using PCR methods with total genomic DNA as the template, some of the cp sequence regions are similar to those in other organelles, thus raising the possibility of 


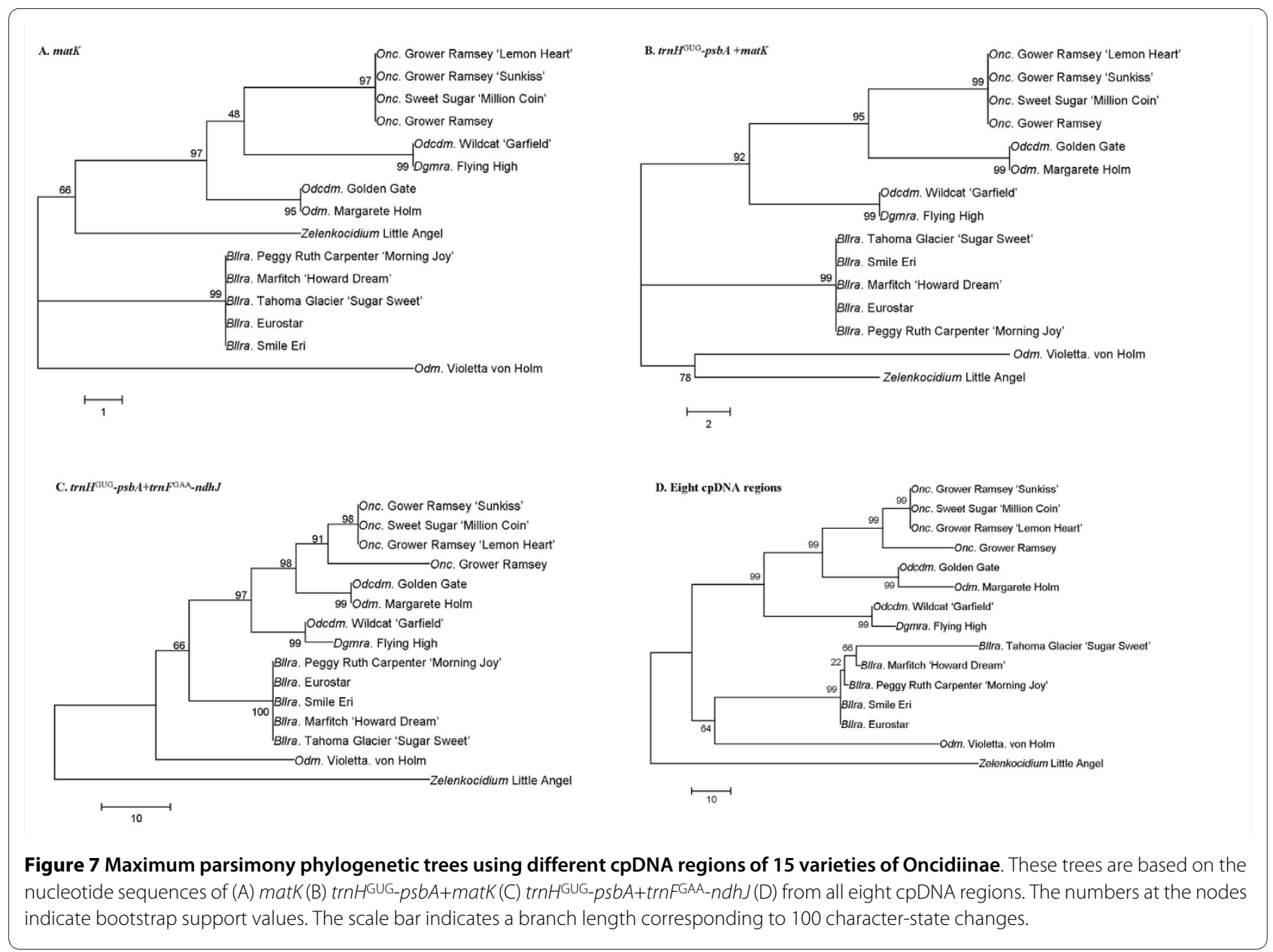

false results. To prevent such results, we used BLAST analysis and different combinations of primers to amplify the same region.

Considerable effort is being expended to investigate phylogenomic relationships among monocots using $\mathrm{cp}$ genomes (see http://www.botany.wisc.edu/monatol/). Here, the phylogenetic position of Orchidaceae among Asparagales is confirmed with the robust support provided by many informative cpDNA characters. Further sampling among orchids in the future phylogenomic studies building on our results will clarify the complex relationships within the large family. Therefore, the cpDNA of Oncidium Gower Ramsey provides valuable information for further orchid $\mathrm{cp}$ genome sequencing and phylogenomics.

\section{ndh genes in Oncidiinae cpDNA}

In higher plant chloroplasts, the $\mathrm{NAD}(\mathrm{P}) \mathrm{H}$ dehydrogenase (NDH) complex functions in PSI cyclic electron flow and chlororespiration [23]. Eleven subunits of the chloroplast $n d h$ genes $(n d h A-n d h K)$ are encoded in the chloroplast genome. In addition 3 cyanobacterial orthologs, nuclear-encoded subunits genes (NdhM-NdhO), have also been identified in chloroplasts [24]. This indicates that nucleus-encoded $n d h$ genes originated in cyanobacteria and were transferred from the chloroplast genome to the nuclear genome during evolution [25]. However, in Onc. Gower Ramsey, out of all the 11 chloroplastencoded $n d h$ genes, only $n d h E$ theoretically translates into a functional protein. This $n d h$ gene truncation and absence was also observed in P. aphrodite [8]. Using a PCR approach to sequence the $n d h$ genes of 15 varieties, we demonstrated that truncation and absence of $n d h$ genes from the cp is a general phenomenon in Oncidiinae.

The loss-of-function of $n d h$ genes or other chloroplastencoded genes occurs in many plants, such as parasitic plants [26-30] and achlorophyllous orchids [31,32]. Lossof-function in $n d h$ genes occurs not only in heterotrophic plants, but also in autotrophic species. In Pinus thunbergii, all $11 n d h$ genes were putative loss-of-function alleles [33], and in another Coniferales species, Keteleeria davidiana, was also found to contain nonfunctional $n d h$ genes [34]. In three Gnetophytes, which comprise three related families of woody gymnosperms (Welwitschia mirabilis, Ephedra equisetina, and Gnetum parvifolium), all $11 \mathrm{ndh}$ 
genes are non-functional, 10 being absent and one, $n d h B$, being a pseudogene $[34,35]$. It is interesting to note that this $n d h$ deletion does not occur in all gymnosperm species. The $n d h$ genes exist in the chloroplast genomes of Cryptomeria and Cycas [36,37]. It is possible that ancestral plastid $n d h$ genes were transferred to the nucleus, remaining functional to this day $[8,33]$.

Loss-of-function $n d h$ genes also occur in other orchids [38]. Phaelenopsis aphrodite lacks the $n d h A, n d h F$, and $n d h H$ genes, and only remnants of the other eight subunits sequences were found [8]. The $11 n d h$ genes were either truncated or frame-shifted, suggesting that they are nonfunctional [8]. In this report, we demonstrated that $n d h$ gene deletion is also common in Oncidiinae: the deletion pattern differs not only between Oncidium and Phalaenopsis (Figure 6), but even within the 15 Oncidiinae species analyzed (Figure 5).

From a physiological view, since parasitic plants obtain organic nutrients from the host, loss of functional $n d h$ genes from the chloroplast is not surprising. However, this does not explain why most $n d h$ genes are non-functional or deleted in autotrophic plants. The presence of $n d h$ homologs encoded within the nucleus was confirmed using PCR assays of total DNA of Phalaenopsis [8]. The resulting sequences are in frame and imply that the ancestral functional $n d h$ copies of the plastid genome may have been transferred to the nuclear genome [8].

\section{Phylogenetic analysis of 15 Oncidium species}

Because it is easy to perform interspecific or intergeneric crosses with orchids, there are many artificial intergeneric hybrids. These hybrids are not distinct phenotypically and are partially named according to their parental background. However, hybrids with different parental backgrounds may be classified into the same genus. In addition, Hybrids from differently named genera may originate from the same female parent. Economic varieties of orchids are generally hybrids of other hybrids and some of the parental information has been lost. To further complicate matters, changes in the names of genera and taxonomy of the Oncidiinae are frequent. In 2004, the names of more than 2200 hybrids comprising some $20 \%$ of the Oncidium group were changed. For example, Colmanara Wildcat was changed to Odcdm. Wildcat and Oncidium Little Angel was changed to Zelenkocidium Little Angel. These changes and whether there were grounds for them could be clarified by looking carefully at the cpDNA, which could identify the female parent.

Among the eight sequences studied here, the phylogenetic analysis using mat $K$ was most well-correlated with the parent relationship (Figure 7A). There are at least three advantages of using the matK region for phylogenetic analysis: (1) this region is variable at the interspecies level [22]; (2) this region is easy to amplify using pub- lished primer sequences [39]; and (3) a large amount of sequence information about Oncidiinae mat $K$ is readily available in the public domain, including the number of sequences (695) and the length of the sequences (791 bp). Here, we performed a phylogenetic analysis by using 15 varieties and their 180 related sequences. Among the results we found several areas of divergence between the taxonomy of Oncidiinae based on morphology and our phylogenetic analyses. For example, the female parent of Beallara is Miltassia, making the grandparent Brassia. The sequences of Beallara were highly correlated with other Brassia species, and most closely with the female parent Brassia verrucosa (Accession no.: EF079203, data not shown). However, the phylogenetic analysis of these sequences showed that the Odontoglossum mat $K$ was dispersed around the Oncidium group (data no shown). Result such as these suggests that analysis of a single region may not contain enough information for interspecies phylogenetic analysis.

To solve this problem, available sequence information must be increased. During phylogenetic analysis, correlation is dependent on the length and properties of DNA or amino acid information. Because the information on orchid cpDNA is limited, the combination of several sequences derived by PCR using universal primers could be a successful strategy [see $[22,31,32,40,41]]$. In this report, eight sequences from each species were combined (total length of $7042 \mathrm{bp}$ ) and were well-correlated with the parent relationship. However, to manage labor and supply costs, we wanted to identify the smallest region that would result in the same performance as using all eight regions. Therefore, we combined divergent cpDNA sequences such as $m a t K$ for further analyses. In addition to $m a t K$, the $t r n H^{G U G}-p s b A$ region is another divergent cpDNA region useful for phylogenetic analysis [21,22]. Various expansions or contractions of inverted repeats (IRs) in chloroplast genomes lead to diverse ${ }^{t} n H^{\mathrm{GUG}_{-}}$ $p s b A$ regions [42-44]. The structural changes in cpDNA provide useful phylogenetic inferences [45]. According to these data, the $\operatorname{trnH}^{\mathrm{GUG}}$ - $p s b A$ regions are informationrich and could be used for phylogenetic analysis.

In addition, $n d h$ gene deletion is a unique feature that may also provide useful information for parentage analysis. The $t r n F^{\mathrm{GAA}}-n d h J-n d h K-n d h C$ region could be amplified by PCR in all of the 15 varieties. Therefore, different combinations of these information-rich regions (matK,

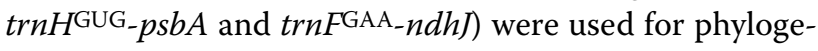
netic analysis. According to our results, two variable cpDNA regions, $t r n H^{\mathrm{GUG}}-p s b A$ and $\operatorname{trn} F^{\mathrm{GAA}}-n d h J$, could provide sufficient information for genus-to-species level phylogenetic analysis.

However, several questions require further investigation. The first is the placement of Odm. Violetta von Holm, whose female parent is Odm. bictoniense. Irrespec- 
tive of the cpDNA template, Odm. Violetta von Holm did not correlate with Odcdm. Golden Gate or Odm. Margarete Holm, which are both derived from the same female parent. The second is the placement of Dgmra. Flying High, which has the female parent Mtssa. Jet Setter. Theoretically, the cpDNA of Dgmra. Flying High should be closely related to Beallara species, which are derived from a Miltassia female parent; however our data indicated that Dgmra. Flying High is more similar to Odontoglossum.

There are many advantages to using cpDNA for phylogenetic and parentage analysis. But this genetic information is only derived from the female parent. Therefore, in the future nuclear genes also need to be analyzed for parentage analysis [46]. In Pleione, the nrITS region was found to be more variable than the plastid regions sequenced, and nrITS gene trees were largely congruent with those inferred from the plastid regions [46]. Our data here suggest that the taxonomy of the Oncidiinae may be improved by both chloroplast and nuclear genome analysis.

\section{Conclusion}

In this report, we used $P$. aphrodite as a prototype to design primers to complete the Onc. Gower Ramsey genome sequence. The primers and the genome sequence information obtained will be useful for further orchid cpDNA sequencing and broad phylogenetic analyses among monocots. Gene annotation showed that most of the $n d h$ genes in Oncidiinae are non-functional, with the exception of $n d h E$, which could theoretically produce a functional protein. In the previous reports, non-functionality of $n d h$ genes has been found in photosynthetic orchids and gymnosperms, such as in Pinus thunbergii and Phalaenopsis. In this report, using a PCR approach, we identified the $n d h$ genes in different Oncidiinae plants. The $n d h$ genes were also non-functional in most of the plants tested, except for $n d h E$ in four Oncidium species and Odm. Margarete Holm. These genes would be useful for parentage analysis. The non-protein coding regions $t r n H^{\mathrm{GUG}_{-}} p s b A$ and $\operatorname{trn} F^{\mathrm{GAA}_{-} n d h J}$ were also determined to be cpDNA regions that would be the most useful for phylogenetic analysis. When these regions were checked in commercial varieties, most confirmed to previously known inheritance information; however, some variations need further investigation. Also, to confirm and complement the results obtained from cpDNA, genetic information may also be derived from nuclear DNA. We conclude that complete chloroplast genome information is useful for plant phylogenetic and evolutionary studies in Oncidium breeding and variety identification.

\section{Additional material}

\author{
Additional file 1 Primers for Oncidium Gower Ramsey chloroplast \\ sequencing. Excel file containing Primers for Oncidium Gower Ramsey \\ chloroplast sequencing.
}

Additional file $\mathbf{2}$ The accession numbers of the Oncidiinae chloroplast sequences for $\boldsymbol{n d h}$ gene and phylogenetic analysis. Excel file containing the accession numbers of the Oncidiinae chloroplast sequences for $n d h$ gene and phylogenetic analysis.

Additional file 3 Maximum parsimony phylogenetic trees using $r b c L$ regions of $\mathbf{1 5}$ varieties of Oncidiinae. These trees are based on the nucleotide sequences of $r b c L$ sequences. The numbers at the nodes indicate bootstrap support values. The scale bar indicates a branch length corresponding to 100 character-state changes.

Additional file 4 Maximum parsimony phylogenetic trees using accD regions of $\mathbf{1 5}$ varieties of Oncidiinae. These trees are based on the nucleotide sequences of $\operatorname{acc} D$ sequences. The numbers at the nodes indicate bootstrap support values. The scale bar indicates a branch length corresponding to 100 character-state changes.

\section{Competing interests}

The authors declare that they have no competing interests.

\section{Authors' contributions}

FHW, MTC, DCL, and CTH performed PCR and primer design. YWL performed the bioinformatic analysis. HD contributed to chloroplast genome annotation, correcting errors in genome sequence, assembling the genome map and participated in manuscript preparation. MRD performed broad phylogenetic analyses and participated in manuscript preparation. CSL conceived this project, supervised PCR, primer design, bioinformatic analysis, and participated in the preparation of the manuscript. All authors read and approved the final manuscript.

\section{Acknowledgements}

We thank Tze-In Yeh for her assistance in PCR. The authors would also like to acknowledge Dr. N.D. Singh in the Daniell lab for drawing the map in Figure 3. This work was supported by the Development Program of Industrialization for Agricultural Biotechnology, Taiwan. Support for MRD was also obtained from the Plant Molecular Biology Center, Northern Illinois University, USA.

\section{Author Details}

${ }^{1}$ Agricultural Biotechnology Research Center, Academia Sinica, Taipei, Taiwan, 2Department of Molecular Biology and Microbiology, College of Medicine, Biomolecular Science Building, University of Central Florida, Orlando, USA and 32Department of Biology, Northern Illinois University, DeKalb, USA

Received: 26 June 2009 Accepted: 16 April 2010

Published: 16 April 2010

\section{References}

1. Webster P: Oncidium subtribe. In The Orchid Genus Book Hunters Breeze, USA; 1992.

2. Chase W, Palmer JD: Chloroplast DNA systematics of lilioid monocots: resources, feasibility, and an example from the Orchidaceae. Amer J Bot 1989, 76:1720-1730.

3. Tsai CC, Huang SC, Huang PL, Chen YS, Chou CH: Phenetic relationship and identification of subtribe Oncidiinae genotypes by random amplified polymorphic DNA (RAPD) markers. Sci Hort 2002, 101:315-325.

4. Tien X, Li DZ: Application of DNA sequences in plant phylogenetic study. Acta Bot Yumnan 2002, 24:170-184.

5. Cao Q-q, Zhen Z, Jiang J, Liu Y-f, Feng Y-q, Qin L: Chloroplast DNA analysis technology and its application in Castanea. J Fruit Sci 2008, 25:396-399.

6. Dhingra A, Folta KM: ASAP: Amplification, sequencing, and annotation of plastomes. BMC Genomics 2005, 6:176.

7. Wu FH, Kan DP, Lee SB, Daniell H, Lee YW, Lin CC, Lin NS, Lin CS: Complete nucleotide sequence of Dendrocalamus latiflorus and Bambusa oldhamii chloroplast genomes. Tree Physiol 2009, 29:847-856. 
8. Chang CC, Lin HC, Lin IP, Chow TY, Chen HH, Chen WH, Chen CH, Liu SM, Chang CC, Lin CY, Chaw SM: The chloroplast genome of Phalaenopsis aphrodite and comparative analysis of evolutionary rate with that of Grasses. Mol Biol Evol 2006, 23:279-291.

9. Daniell H, Singh ND, Mason H, Streatfield SJ: Plant-made vaccine antigens and biopharmaceuticals. Trends Plant Sci 2009, 14:669-679.

10. Verma D, Daniell H: Chloroplast vector systems for biotechnology applications. Plant Physiol 2007, 145:1129-1143.

11. Sheu JJ, Yu TS, Tong WF, Yu SM: Carbohydrate starvation stimulates differential expression of rice a-amylase genes that is modulated through complicated transcriptional and postranscriptional process. $J$ Biol Chem 1996, 27:26998-27004

12. Cai Z, Penaflor C, Kuehl J, Leebens-Mack J, Carlson J, dePamphilis C, Boore $J$, Jansen R: Complete chloroplast genome sequences of Drimys, Liriodendron, and Piper: Implications for the phylogeny of magnoliids. BMC Evol Biol 2006, 6:77.

13. Goremykin VV, Hirsch-Ernst KI, Wölfl S, Hellwig FH: Analysis of the Amborella trichopoda chloroplast genome sequence suggests that Amborella is not a basal angiosperm. Mol Biol Evol 2003, 20:1499-1505.

14. Hansen DR, Dastidar SG, Cai Z, Penaflor C, Kuehl JV, Boore JL, Jansen RK: Phylogenetic and evolutionary implications of complete chloroplast genome sequences of four early-diverging angiosperms: Buxus (Buxaceae), Chloranthus (Chloranthaceae), Dioscorea (Dioscoreaceae), and Illicium (Schisandraceae). Mol Phylogenet Evol 2007, 45:547-563.

15. Leebens-Mack J, Raubeson LA, Cui L, Kuehl JV, Fourcade MH, Chumley TW, Boore JL, Jansen RK, dePamphilis CW: Identifying the basal angiosperm node in chloroplast genome phylogenies: sampling one's way out of the Felsenstein zone. Mol Biol Evol 2005, 22:1948-1963.

16. Zwickl DJ: Genetic algorithm approaches for the phylogenetic analysis of large biological sequence datasets under the maximum likelihood criterion. In Ph.D. dissertation The University of Texas at Austin; 2006.

17. Swofford DL: PAUP*: Phylogenetic analysis using parsimony (*and other methods). In Version 4 Sinauer Associates, Sunderland, MA; 2003.

18. Felsenstein J: Confidence limits on phylogenies: an approach using the bootstrap. Evolution 1985, 39:783-791.

19. Wyman SK, Jansen RK, Boore JL: Automatic annotation of organellar genomes with DOGMA. Bioinformatics 2004, 20:3252-3255.

20. Tamura K, Dudley J, Nei M, Kumar S: MEGA 4: molecular evolutionary genetics analysis (MEGA) software Version 4.0. Mol Biol Evol 2007, 24:1596-1599.

21. Kresss WJ, Wurdack KJ, Zimmer EA, Weigt LA, Janzen DH: Use of DNA barcodes to identify flowering plants. Proc Natl Acad Sci USA 2005, 102:8369-8374

22. Lahaye R, Bank M van der, Bogarin B, Warner J, Pupulin F, Gigot G, Maurin O, Duthoit S, Barraclough TG, Savolainen V: DNA barcoding the floras of biodiversity hotspots. Proc Natl Acad Sci USA 2008, 105:2923-2928.

23. Sazanov LA, Burrows PA, Nixon PJ: The plastid $n d h$ genes code for an $\mathrm{NADH}$-specific dehydrogenase: Isolation of a complex I analogue from pea thylakoid membranes. Proc Natl Acad Sci USA 1998, 95:1319-1324.

24. Rumeau D, Bécuwe-Linka N, Beyly A, Louwagie M, Garin J, Peltier G: New subunits $\mathrm{NDH}-\mathrm{M},-\mathrm{N}$, and $-\mathrm{O}$, encoded by nuclear genes, are essential for plastid Ndh complex functioning in higher plants. Plant Cell 2005, 7:219-232.

25. Muraoka R, Okuda K, Kobayashi Y, Shikanai T: A eukaryotic factor required for accumulation of the chloroplast $\mathrm{NAD}(\mathrm{P}) \mathrm{H}$ dehydrogenase complex in Arabidopsis. Plant Physio/ 2006, 142:1683-1689.

26. dePamphlis CW, Palmer JD: Loss of photosynthetic and chlororespiratory genes from the plastid genome of a parasitic flowering plant. Nature 1990, 348:337-339.

27. Wickett NJ, Zhang Y, Hansen SK, Roper JM, Kuehl JV, Plock SA, Wolf PG, dDePamphilis CW, Boore JL, Goffinet B: Functional gene losses occur with minimal size reduction in the plastid genome of the parasitic liverwort Aneura mirabilis. Mol Biol Evol 2008, 25:393-401.

28. Haberhausen $\mathrm{G}$, Zetsche $\mathrm{K}$ : Functional loss of $n$ dh genes in an otherwise relatively unaltered plastid genome of the holoparasitic flowering plant Cuscuta reflexa. Plant Mol Biol 1994, 24:217-222.

29. McNeal JR, Kuehl JV, Boore JL, dePamphilis CW: Complete plastid genome sequences suggest strong selection for retention of photosynthetic genes in the parasitic plant genus Cuscuta. BMC Plant Biol 2007, 7:57.
30. Revill MJ, Staney S, Hibberd JM: Plastid genome structure and loss of photosynthetic ability in the parasitic genus Cuscuta. J Exp Bot 2005, $56: 2477-2486$

31. Cameron KM: Utility of plastid $p s a B$ gene sequences for investigating intrafamilial relationships within Orchidaceae. Mol Phylog Evol 2004, 31:1157-1180

32. Cameron KM, Molina C: Photosystem II gene sequences of $p s b B$ and $p s b C$ clarify the phylogenetic position of Vanilla (Vanilloideae, Orchidaceae). Cladistics 2006, 22:239-248.

33. Wakasugi T, Tsudzuki J, Ito S, Nakashima K, Tsudzuki T, Sugiura M: Loss of all $n d h$ genes as determined by sequencing the entire chloroplast genome of the black pin Pinus thunbergii. Proc Natl Acad Sci 1994, 91:9794-9798.

34. Wu CS, Lai YT, Lin CP, Wang YN, Chaw SM: Evolution of reduced and compact chloroplast genomes (cpDNAs) in gnetophytes: selection toward a lower-cost strategy. Mol Phylog Evol 2009, 52:115-124.

35. McCoy SR, Kuehl JV, Boore JL, Raubeson LA: The complete plastid genome sequence of Welwitschia mirabilis: an unusually compact plastome with accelerated divergence rates. BMC Evol Biol 2008, 8:130

36. Hirao T, Watanabe A, Kurita M, Kondo T, Takata K: Complete nucleotide sequence of the Cryptomeria japonica D Don chloroplast genome and comparative chloroplast genomics: diversified genomic structure of coniferous species. BMC Plant Biol 2008, 8:70.

37. Wu CS, Wang YN, Liu SM, Chaw SM: Chloroplast genome (cpDNA) of Cycas taitungensis and $56 \mathrm{cp}$ protein-coding genes of Gnetum parvifolium: insights into cpDNA evolution and phylogeny of extant seed plants. Mol Biol Evol 2007, 24:1366-137.

38. Neyland R, Urbatsch LE: The $n d h F$ chloroplast gene detected in all vascular plant divisions. Planta 1996, 200:273-277.

39. Cuenoud P, Savolainen V, Chatrou LW, Powell M, Grayer RJ, Chase MW: Molecular phylogenetics of Caryophyllales based on nuclear 18S rDNA and plastid $r b c L$, atpB, and matK DNA sequences. Amer J Bot 2002, 89:132-144.

40. Freudenstein JV, Berg C van den, Goldman DH, Kores PJ, Molvray M, Chase MW: An expanded plastid DNA phylogeny of Orchidaceae and analysis of Jackknife branch support strategy. Amer J Bot 2004, 91:149-157.

41. Bellusci F, Pellegrino G, Palermo AM, Musacchio A: Phylogenetic relationships in the orchid genus Serapias $L$ based on noncoding regions of the chloroplast genome. Mol Phylogenet Evol 2008, 47:986-991.

42. Palmer JD, Stein DB: Conservation of chloroplast genome structure among vascular plants. Curr Genet 1986, 10:823-833.

43. Plunkett GM, Downie SR: Expansion and contraction of the chloroplast inverted repeat in Apiaceae subfamily Apioideae. Syst Bot 2000, 25:648-667.

44. Wang RJ, Cheng CL, Chang CC, Wu CL, Su TM, Chaw SM: Dynamics and evolution of the inverted repeat-large single copy junctions in the chloroplast genomes of monocots. BMC Evol Biol 2008, 8:36.

45. Raubeson LA, Jansen RK: Chloroplast genomes of plants. In Plant Diversity and Evolution: Genotypic and phenotypic variation in higher plants Edited by: Henry RJ. Wallingford: CABI Publishing; 2005:45-68.

46. Gravendeel B, Eurlings MCM, Berg C van den, Cribb PJ: Phylogeny of Pleione (Orchidaceae) and parentage analysis of its wild hybrids based on plastid and nuclear ribosomal ITS sequences and morphological data. Syst Bot 2004, 29:50-63.

doi: $10.1186 / 1471-2229-10-68$

Cite this article as: Wu et al., Complete chloroplast genome of Oncidium Gower Ramsey and evaluation of molecular markers for identification and breeding in Oncidiinae BMC Plant Biology 2010, 10:68 